\title{
Efflux only impacts drug accumulation in actively growing cells
}

\author{
Emily E Whittle ${ }^{1}$, Helen E McNeil ${ }^{1}$, Eleftheria Trampari' ${ }^{2}$, Mark Webber ${ }^{2}$, Tim W \\ Overton $^{3}$, Jessica M A Blair ${ }^{*}$
}

${ }^{1}$ College of Medical and Dental Sciences, Institute of Microbiology and Infection, University of Birmingham, Birmingham, UK

${ }^{2}$ Quadram Institute Bioscience, Norwich Research Park, Norwich, United Kingdom

${ }^{3}$ School of Chemical Engineering, University of Birmingham, Birmingham, UK.

\section{*Correspondence:}

Jessica M A Blair

J.M.A.Blair@bham.ac.uk

\section{Abstract}

For antibiotics with intracellular targets, effective treatment of bacterial infections requires the drug to accumulate to a high concentration inside cells. Bacteria produce a complex cell envelope and possess drug-export efflux pumps to limit drug accumulation inside cells. Decreasing cell envelope permeability and increasing efflux pump activity can reduce intracellular accumulation of antibiotics, and are commonly seen in antibiotic resistant strains. Here, we show that the balance between influx and efflux differs depending on bacterial growth phase in Gramnegative bacteria. Accumulation of the model fluorescent drug, ethidium bromide (EtBr) was measured in S. Typhimurium SL1344 (wild-type) and efflux deficient ( $\triangle a c r B)$ strains during growth. In SL1344, EtBr accumulation remained low, 
regardless of growth phase and did not correlate with acrAB transcription. EtBr accumulation in $\triangle a c r B$ was high in exponential phase but dropped sharply later in growth, with no significant difference to SL1344 in stationary phase. Low EtBr accumulation in stationary phase was not due to the upregulation of other efflux pumps, but instead, due to decreased permeability of the envelope in stationary phase. RNAseq identified changes in expression of several pathways that remodel the envelope in stationary phase, leading to lower permeability. This study shows that efflux is only important for maintaining low drug accumulation in actively growing cells, and that envelope permeability is the predominant factor dictating the rate of drug entry in stationary phase cells. This conclusion means that (i) antibiotics with intracellular targets may be less effective in complex non-growing or slow-growing bacterial infections where intracellular accumulation may be low, (ii) efflux inhibitors may be successful in potentiating the activity of existing antibiotics, but potentially only for bacterial infections where cells are actively growing and (iii) the remodelling of the cell envelope prior to stationary phase could provide novel drug targets. 


\section{Introduction}

2 Antibiotic treatment failure in clinical infections is increasingly common due to the

3 rise in multi-drug resistant (MDR) Gram-negative bacteria. Gram-negative infections

4 are particularly difficult to treat due to their impermeable outer membranes and efflux

5 pumps which actively export antibiotic molecules out of the bacterial cell. Successful

6 treatment relies on high concentrations of antibiotic accumulating within bacterial

7 cells, which is a function of antibiotic influx and the rate of antibiotic efflux ${ }^{1}$.

8 Small hydrophilic antibiotics such as $\beta$-lactams enter a Gram-negative bacterial cell

9 through membrane pores called porins. The major porins of Enterobacteriaceae are

$10 \mathrm{OmpF}$ and $\mathrm{OmpC}^{2}$. Downregulation of porin genes contributes to antibiotic

11 resistance by preventing antibiotics entering the cell ${ }^{3}$. In addition, mutations in the

12 porin protein which change the channel diameter ${ }^{4,5}$ or the electric field inside the

13 porin can block translocation of drugs across the membrane ${ }^{5}$.

14 Some drugs can enter Gram-negative cells through the lipid outer and inner

15 membranes via 'self-promoted uptake'. This mechanism has been described for

16 EDTA, Polymyxin B, colistin and other cationic antimicrobial peptides (CAMPs), and

17 aminoglycoside antibiotics ${ }^{6-8}$. The chelator, EDTA, acts as a permeabiliser by

18 displacing and chelating the cations $\left(\mathrm{Mg}^{2+}\right.$ or $\left.\mathrm{Ca}^{2+}\right)$ that are essential for the stability

19 of LPS and the $\mathrm{OM}^{6,9}$. CAMPs interact with anionic groups on lipid A, breaching the

20 outer membrane, and porate in the inner membrane, leading to bacterial death.

21 Enterobacteriaceae contain efflux pumps from 6 classes. MFS, SMR, MATE, RND

22 and the recently described PACE pumps ${ }^{10}$ utilise the proton motive force for export

23 of molecules such as antibiotics, and ABC (ATP binding cassette) pumps utilise ATP 
24 hydrolysis. Resistance-Nodulation-Division (RND) pumps are commonly upregulated

25 in clinical isolates and can contribute to resistance to a number of antibiotic classes,

26 as well as dyes, detergents and biocides ${ }^{11}$. The best described RND pump is AcrAB-

27 TolC, found in Salmonella enterica serovar Typhimurium (S. Typhimurium) and E.

28 coli. As efflux pumps underpin antibiotic resistance in essentially all bacteria of

29 clinical and veterinary importance ${ }^{12,13}$ there is ongoing active research into the

30 development of efflux inhibitors to potentiate the action of existing antibiotics.

31 Previous studies undertaken with cells in exponential growth phase have highlighted

32 the importance of efflux pumps in minimising intracellular drug accumulation ${ }^{14-17}$.

33 However, transcription of $a c r A B$ is growth phase dependent, with a peak in mid-

34 exponential phase, which drops as cells enter into stationary phase ${ }^{18}$. The

35 importance of AcrAB-TolC in bacterial cells in stationary phase which are slow-

36 growing or non-growing is not known. However, it has been suggested that whereas

37 survival of exponential-phase $E$. coli following treatment with the anionic detergent

38 sodium dodecyl sulphate (SDS) is dependent on efflux, stationary phase cell survival

39 is efflux-independent and rather is mediated by decreased permeability of the

40 bacterial cell envelope, directed by the stationary phase sigma factor $\operatorname{RpoS}^{19}$. Little is

41 known about the balance between influx and efflux in different growth phases and

42 how this may relate to different growth states that may occur in an infection.

43 Previous studies have shown that the E. coli envelope changes in stationary phase

44 when compared to logarithmic growth and it is possible that this could alter antibiotic

45 influx in non-growing bacterial cells. Outer membrane changes include a decrease in

46 the overall concentration of membrane proteins ${ }^{20}$ and an increase in lipoprotein

47 crosslinked to peptidoglycan ${ }^{21,22}$ to strengthen the outer barrier. In the inner 
48 membrane, the composition of fatty acids changes with a decrease in

49 monounsaturated fatty acids ${ }^{23}$ and an increase in cyclopropane fatty acids, catalysed

50 by $\mathrm{Cfa}^{24}$. Increased layers of peptidoglycan have also been described in stationary

51 phase $^{25}$

52 Using a combination of fluorescent drug accumulation assays ${ }^{17}$, and measurement

53 of efflux gene transcription in wild-type and efflux mutant strains we here assess the

54 importance of the balance between influx and efflux in different growth phases in

55 Gram-negative bacteria, using the model organism Salmonella enterica serovar

56 Typhimurium. We also use RNASeq to measure the global transcriptome as bacteria

57 enter stationary phase and correlate transcriptomic changes with biochemical and

58 physiological changes in the cell envelope that lead to alterations in permeability.

59 Results

60 Accumulation level of drugs by $S$. Typhimurium is independent of growth

61 phase-dependent acrAB transcription

62 Using a recently developed flow cytometry method ${ }^{17}$, both the intracellular

63 accumulation of the fluorescent dye ethidium bromide (EtBr), and the transcription of

$64 a c r A B$ (via a promoter-GFP fusion) were measured in parallel in single cells of

65 Salmonella grown in drug free media. Samples were taken hourly during batch

66 culture before EtBr was added to the sample immediately prior to flow cytometry

67 analysis to measure accumulation ( $\mathrm{EtBr}$ was not present in growing the culture). 


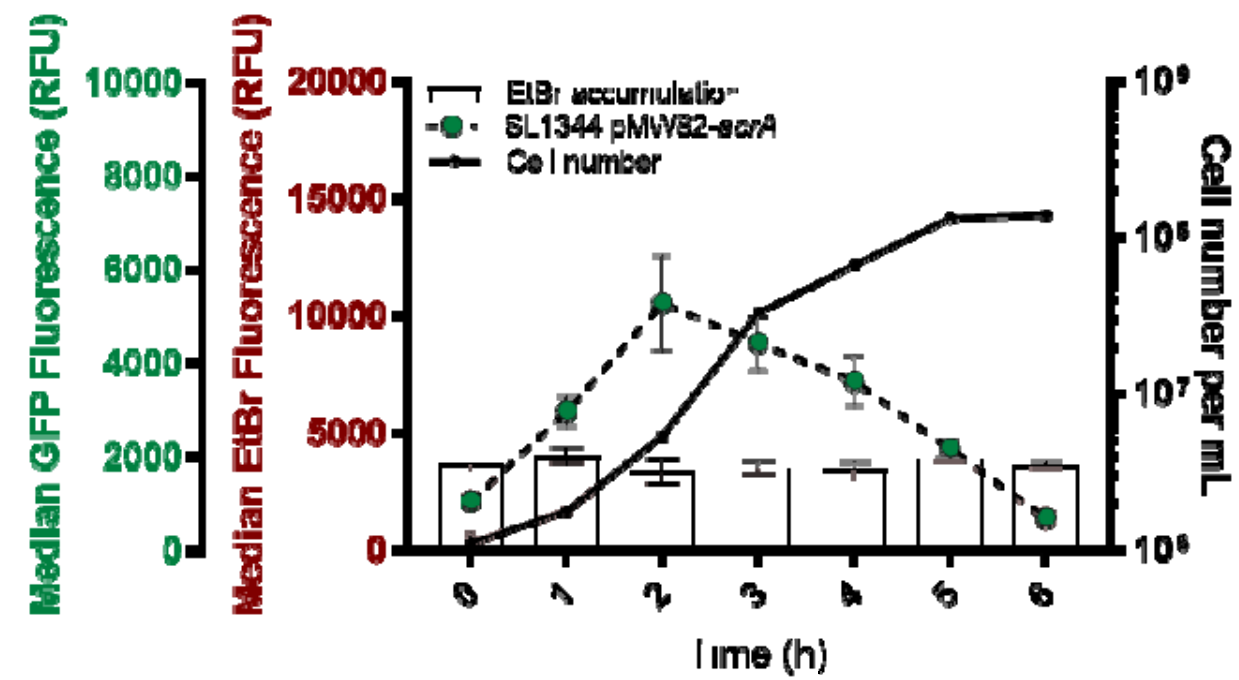

69 Figure 1 Ethidium bromide accumulation and acrAB expression in single cells of S. Typhimurium SL1344

70 across the growth phase. Cell number per $\mathrm{mL}$ was measured in each sample (black lines, numbers indicated

71 on the right $y$-axis). Pink bars indicate median ethidium bromide fluorescence per cell (relating to left red $y$-axis)

72 and dashed lines with green circles show $a c r A B$ expression (median GFP fluorescence per cell from reporter,

73 relating to left green y-axis. All data points are median values from measurements of 10,000 single cells of

74 SL1344. Error bars indicate standard error of the mean (+/- SEM).

75 Transcription of acrAB in SL1344 was growth phase dependent and peaked in early-

76 mid log phase before decreasing towards stationary phase (Figure 1), as previously

77 described ${ }^{18}$. Previous studies have shown that increased expression of acrAB in

78 clinical isolates leads to decreased susceptibility to antibiotics ${ }^{12}$. Given the known

79 role of efflux pumps in drug export, one might predict that EtBr accumulation would

80 be lowest when efflux expression was highest. Our data however show that this is

81 not the case. In SL1344 cells, accumulation of EtBr was low and remained

82 unchanged across growth despite changes in acrAB transcription (Fig 1). Therefore,

83 changes in efflux pump transcription in different growth phases does not alter levels

84 of drug accumulation within the cell.

85 Growth phase-dependent transcription of $\operatorname{acr} A B$ does not correlate with drug 
87 Having shown that acr $A B$ transcription does not correlate with ethidium bromide

88 accumulation, the efflux function in a population of cells was measured to determine

89 whether efflux activity varied with growth phase (and acrAB expression), even if drug

90 accumulation did not.

91 To measure functional efflux capacity of cells we used the previously described

92 direct efflux activity assay ${ }^{14}$ which was further optimised to analyse efflux capacity at

933 different time points across growth in SL1344. This assay determines the efflux

94 capacity of the cell based on the activity of all efflux pumps (not just AcrAB-TolC)

95 that are able to transport EtBr. Cultures grown for 1, 3 and 5 hours had the same

96 capacity to efflux the substrate as there was no significant difference in efflux rate

97 between samples taken at each time point (Figure 2A) (based on time taken for

98 ethidium bromide fluorescence to drop 10\%, $25 \%$ and $50 \%$ from its maximum

99 fluorescence value) regardless of the different levels of acr $A B$ transcription at these

100 time points already established.

A

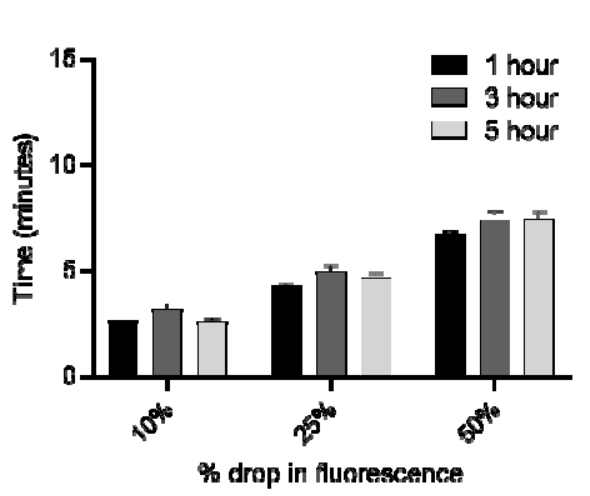

B

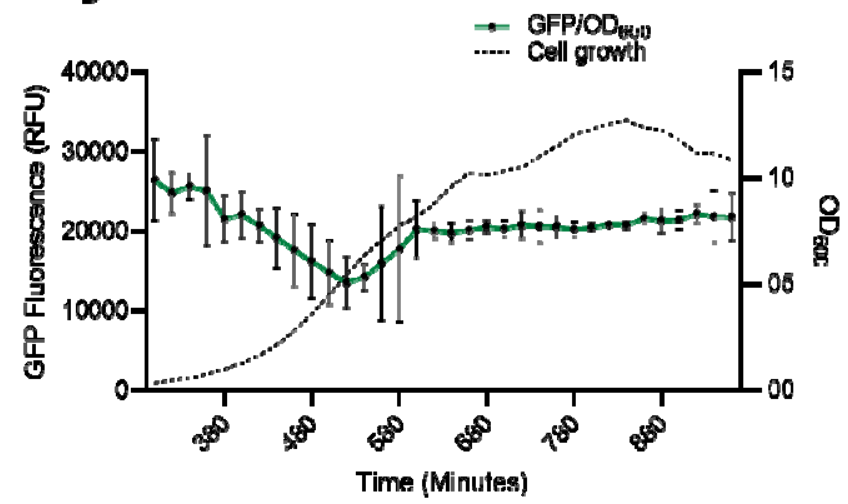

Figure 2 (A)Time taken for ethidium bromide to be removed from SL1344 cells at 1,3 and 5 hours. Bars represent the time taken for ethidium bromide fluorescence to drop $10 \%, 25 \%$ and $50 \%$ from its original value. Data is based on 3 biological replicates with error bars showing standard error of the means (SEM). 1 hour (black), 3 hours (dark grey) and 5 hours (light grey) are shown. There was no significant difference in the time taken to export EtBr at each time point. (B) GFP/OD 600 from SL1344 AcrB-GFP over 16 hours of growth in MOPs minimal media. This graph shows GFP/OD 600 from AcrB-GFP at the end of lag phase (300 minutes) until the last time point at 16 hours. The dashed black line shows the $\mathrm{OD}_{600}$ whereas the green line error bars $+/-\mathrm{SEM}$ shows GFP fluorescence. SL1344 autofluorescence was subtracted from this data. 
110 Taken together, the low accumulation and similar rate of efflux of EtBr across time in

111 SL1344 suggests that although acrAB transcription peaks in mid-exponential phase,

112 activity of the assembled AcrAB-TolC complex remains constant. The AcrB protein is

113 known to be very stable once made with a predicted half-life of 6 days ${ }^{26}$. To measure

114 AcrB protein level at different points during growth a strain was constructed in which

115 the AcrB protein was tagged with GFP at the C-terminus as previously described ${ }^{27}$.

116 The generation time and efflux level in this strain were unaffected confirming that

117 tagging GFP to the C-terminus of AcrB did not affect its function. Measurement of

118 GFP fluorescence during 16 hours of growth showed that AcrB level remains

119 constant (Figure 2B). These data suggest that efflux capacity is constant regardless

120 of growth phase due to the constant level of AcrAB protein within a population and

121 may explain why EtBr accumulation remained low in stationary phase despite

122 decreased efflux gene transcription.

\section{Drug accumulation is only dependent on efflux in actively growing cells}

124 To further dissect the importance of efflux during different growth stages we

125 measured EtBr accumulation (as in Fig. 1) in the presence or absence of AcrAB-

126 TolC function (using SL1344 $\Delta a c r B$ ). The previous results suggest AcrAB-TolC

127 activity is constant, therefore by removing the efflux pump, it was assumed that $\mathrm{EtBr}$

128 accumulation would be high across growth. When measuring EtBr accumulation in

$129 \mathrm{SL} 1344 \Delta \mathrm{acr} B$, after 1 hour of growth, EtBr accumulation was 6-fold higher than in

130 SL1344. This is similar to the growth time point used in most other published studies

131 that have shown an increase in accumulation upon deletion of $\operatorname{acr} B^{11,14,15,17}$.

132 However, EtBr accumulation then decreased dramatically and was not significantly

133 different from WT from 3-6 hours of growth (Figure 3A). This suggests that low 
134 accumulation at 1 hour in SL1344 greatly depends on efflux to export ethidium

135 bromide from actively growing cells. As there is no significant difference between

$136 \Delta a c r B$ and WT cells from 3-6 hours, it suggests that AcrAB-TolC is not important in

137 maintaining low accumulation in slower growing or stationary phase cells. This is

138 also supported by the $a c r A B$ expression data which shows highest expression in the

139 early stages of logarithmic growth. 
A

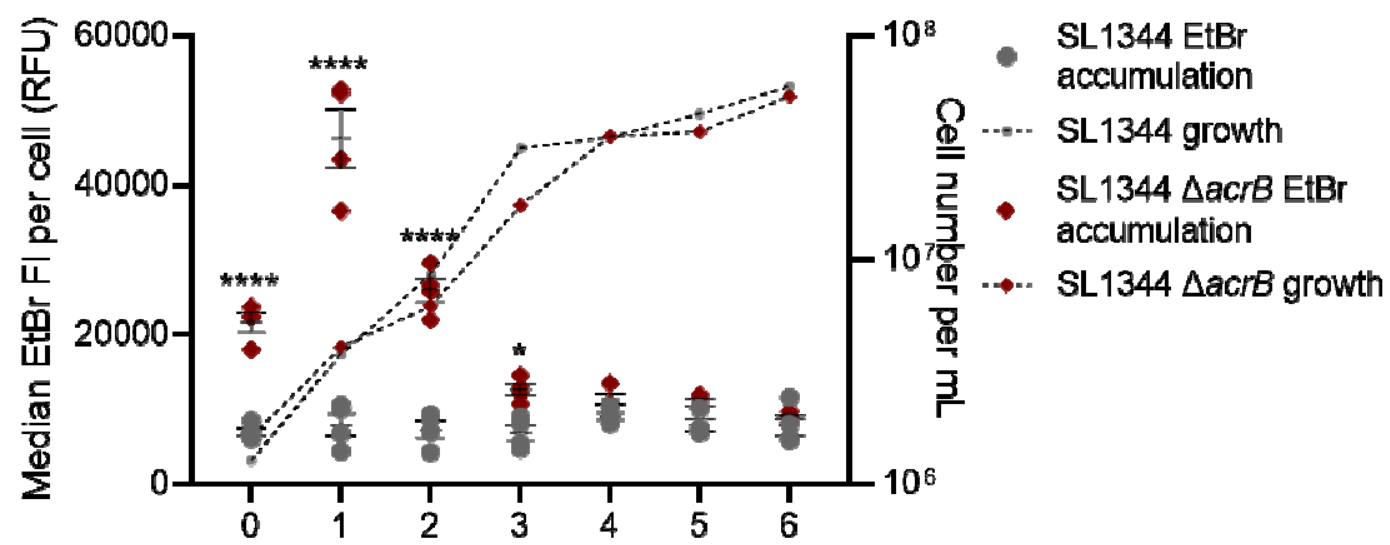

B Time (h)
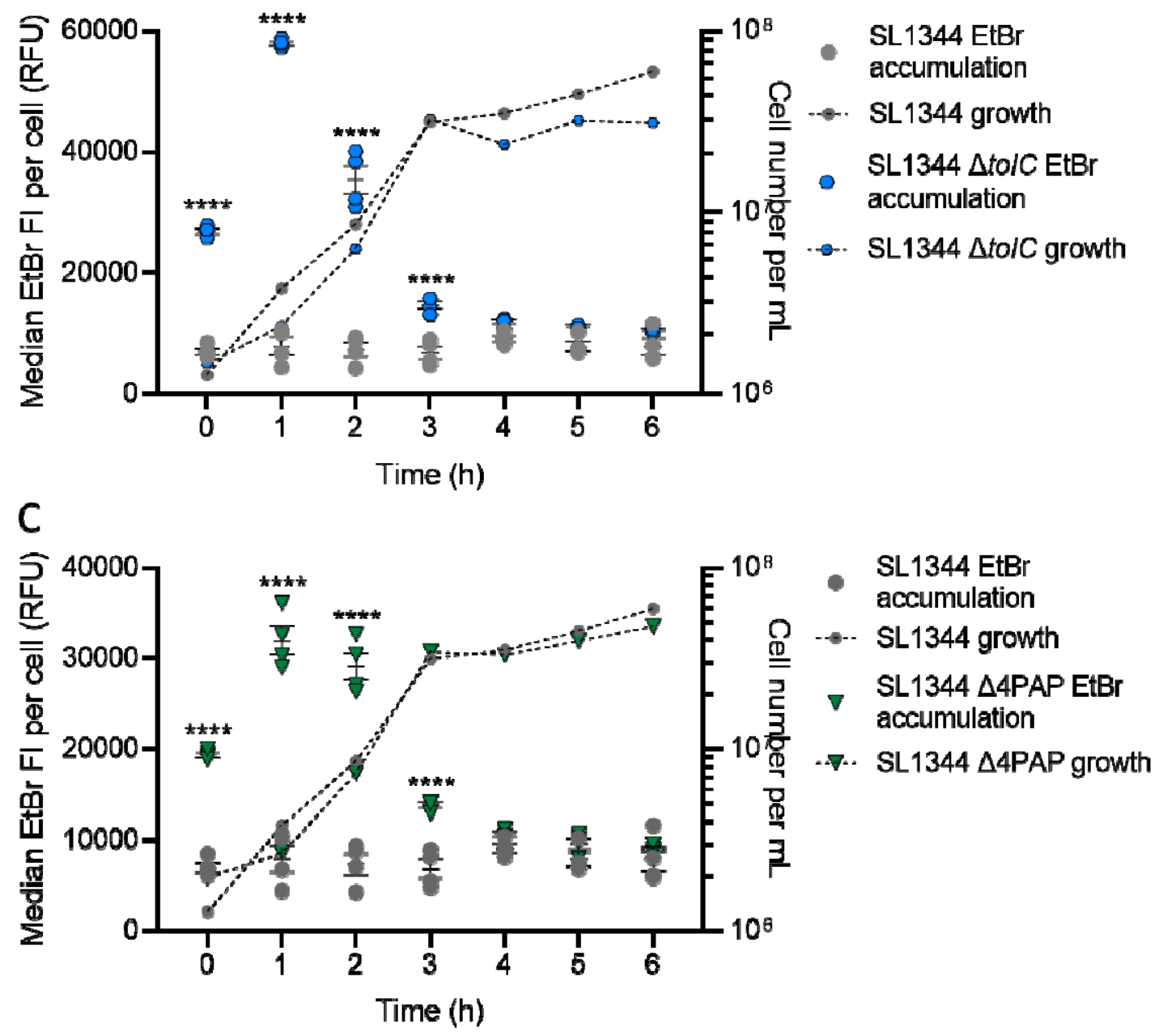

Figure $3 \mathrm{EtBr}$ accumulation in SL1344 and SL1344 $\triangle \mathrm{acrB}(\mathrm{A}), \Delta t o / C$ (B) and $\triangle 4 \mathrm{PAP}$ (C). For each strain the median EtBr fluorescence per cell in 10,000 single cells was measured every hour between 0 and 6 hours of growth for SL1344 (Grey circles) and (A) SL1344 $\triangle a c r B$ (red diamonds) (B) $\Delta$ tolC (blue hexagons) and (C) $\triangle 4 \mathrm{PAP}(\triangle a c r A \triangle a c r E \Delta m d s A \Delta m d t A$ ) (green triangles). Data from 4 biological replicates for each strain are 
the left $y$-axis. Calculated cell number per $\mathrm{mL}$ values were plotted on the right $\mathrm{y}$-axis with corresponding symbols equating to strain and a dashed line to show growth of the cultures. Cell numbers were based on the mean of the same biological replicates and the same gated population that EtBr fluorescence was measured from. Two-way ANOVA and Sidak's multiple comparison test were carried out for statistical analysis. At 0, 1 and 2 hours, EtBr

150 accumulation was significantly increased in $\triangle a c r B$ with $p$ values of $\left.<0.0001{ }^{(* * *}\right)$. .At $0,1,2$ and 3 hours, EtBr

151 accumulation was significantly increased in $\triangle$ tolC and $\triangle 4$ PAP with $p$ values of $<0.0001$.

152 To confirm that low EtBr accumulation in stationary phase was not due to the activity

153 of other RND efflux pumps present in SL1344, EtBr accumulation was also

154 measured in two other mutants of SL1344. In the first, tolC was deleted which

155 compromises most efflux systems in Salmonella which use TolC as a common outer

156 membrane channel. The second strain used lacked all four periplasmic adaptor

157 proteins ( $\triangle 4 \mathrm{PAP} ; \Delta \operatorname{acr} A \Delta \operatorname{acr} E \Delta m d s A \Delta m d t A$ ) and is incapable of assembling any

158 functional RND efflux systems. In both strains, the EtBr accumulation pattern

159 observed recapitulated that seen in SL1344 $\triangle a c r B$, with a peak in accumulation at 1

160 hour, but no significant difference to SL1344 in stationary phase cells (Figure

161 3B\&C). This result showed that low accumulation in stationary phase was not due to

162 any RND pump in SL1344, (nor the ABC pump MacAB-TolC). In addition, we also

163 showed that, apart from $a c r A B$ whose transcription was highest in mid-log phase and

164 lowest in stationary phase, no other RND pump was actively transcribed in the

165 conditions used to measure accumulation capacity across growth (Figure S1). For

166 pumps from other families, only macA (ABC), mdfA (MFS) and mdtK (MATE) were

167 transcribed and only at low levels (Figure S1).

168 Further investigation into the role of efflux pumps in stationary phase $\mathrm{EtBr}$

169 accumulation was carried out by measurement in the presence of the proton motive

170 force inhibitor CCCP. Inhibiting the proton motive force, inhibits the activity of the

171 RND, MFS and MATE pumps of SL1344 ${ }^{28,29}$. In SL1344 in the presence of CCCP,

172 EtBr accumulation peaked at 1 hour (Figure S2). Accumulation levels started to drop

173 into stationary phase, strikingly similar to SL1344 $\triangle a c r B$, again suggesting that low 
174 accumulation in stationary phase is not dependent on RND, MFS or MATE-mediated

175 efflux. This independent confirmation using different mutants and inhibitors

176 demonstrates that the observed low EtBr accumulation in stationary phase is efflux-

177 independent.

178 To investigate whether this was just a Salmonella phenomenon, EtBr accumulation

179 was measured in wild-type and a mutant lacking major RND efflux pump of other

180 Gram-negative bacterial species including Escherichia coli (MG1655 and MG1655

$181 \Delta a c r B)$, Pseudomonas aeruginosa (PA01 and PA01 $\Delta m e x A)$ and Klebsiella

182 pneumoniae (ecl8 and ecl8 acrB::Gm). In E. coli and K. pneumoniae, EtBr

183 accumulation was low throughout growth for the wild-type but peaked at 1 hour for

184 each acr $B$ mutant (Figure S3) and in $P$. aeruginosa, the mex $A$ mutant peaked at 2

185 hours (Figure S4) and then dropped to WT levels in stationary phase. Therefore,

186 very similar observations are seen in a wide range of Gram-negative organisms.

187 The EtBr accumulation pattern in Salmonella was also shown in MOPS minimal

188 medium suggesting that the pattern was not influenced by media type and

189 specifically was not a result of the limitations of LB $^{30}$ (Figure S5). Even though is a

190 well-established and studied model efflux substrate, to counter the possibility that

191 EtBr would give abnormal results which are not representative of other efflux

192 substrates, the same accumulation pattern in Salmonella WT and $\Delta$ tolC was also

193 shown using the lipophilic dye Nile Red (Figure S6). Unlike EtBr, Nile Red does not

194 fluoresce on intercalation with DNA, but rather when bound to phospholipids or

195 triglycerides ${ }^{31}$ showing this is not an artefact of the dye initially used. Together this

196 data shows that the accumulation pattern described in the absence of efflux is

197 consistent regardless of Gram-negative species, media type or efflux substrate used. 
198 Drug accumulation in stationary phase is controlled by reduced membrane permeability

200 Together this data shows that cells from later growth phases minimise intracellular

201 accumulation of $\mathrm{EtBr}$ (and other substrates) in an efflux independent manner. We

202 hypothesised this could be due to a shift in the balance between influx and efflux

203 over growth, with influx rate, controlled by reduced permeability of the outer

204 membrane, being more important in slower growing or stationary phase cells.

205 Several dyes that are often used to probe the permeability of the outer membrane,

206 such as NPN (1-N-Phenylnaphthylamine), are efflux substrates and therefore

207 assessing membrane permeability in strains lacking efflux pumps is problematic.

208 Most hydrophilic antibiotics enter Gram-negative bacterial cells through outer

209 membrane porins such as OmpC and OmpF. To investigate whether porins altered

210 the accumulation of EtBr, accumulation assays were performed using SL1344

211 mutants; $\Delta o m p C / \Delta o m p F / \Delta a c r B, \Delta o m p C / \Delta a c r B, \Delta o m p F / \Delta a c r B, \Delta o m p C$, and $\Delta o m p F$

212 and showed that none had a significantly different EtBr accumulation pattern to those

213 previously seen, confirming that $\mathrm{EtBr}$ doesn't enter $S$. Typhimurium through OmpF or

214 OmpC (Figure S7). A similar observation was made by Murata et al. in E. coli K-12

$213^{32}$, who concluded that the OM bilayer is the predominant mode of EtBr entry.

216 Since SYTO 84 is used in our flow cytometry assay as a probe to stain cells, the

217 accumulation of this dye was first investigated to assess permeability and to

218 determine if it is an efflux substrate. SYTO 84 is marketed as a cell-permeant DNA

219 dye and so is expected to readily enter bacteria. There was no significant difference

220 between the accumulation of SYTO 84 in SL1344 and SL1344 $\triangle a c r B$ and in both

221 strains accumulation peaked after 1 hour of growth (Figure 4). This shows that 
222 SYTO 84 is not an efflux substrate and demonstrates the importance of efflux in

223 maintaining low accumulation of drugs and dyes that are substrates in actively

224 growing cells. However, SYTO 84 fluorescence decreased significantly in both

225 strains on entrance to stationary phase. This suggests that a compound that is not

226 exported via efflux, is also less able to enter bacteria during stationary phase and we

227 hypothesise this is due to a strengthening of the permeability barrier. It is important

228 to note that, although the SYTO 84 fluorescence does reduce around 2.5 -fold in

229 stationary phase, the lowest value is still over 45,000 RFU, so the reduction does not

230 compromise its use to differentiate cells from acellular particles in the $\mathrm{EtBr}$

231 accumulation assays using flow cytometry.

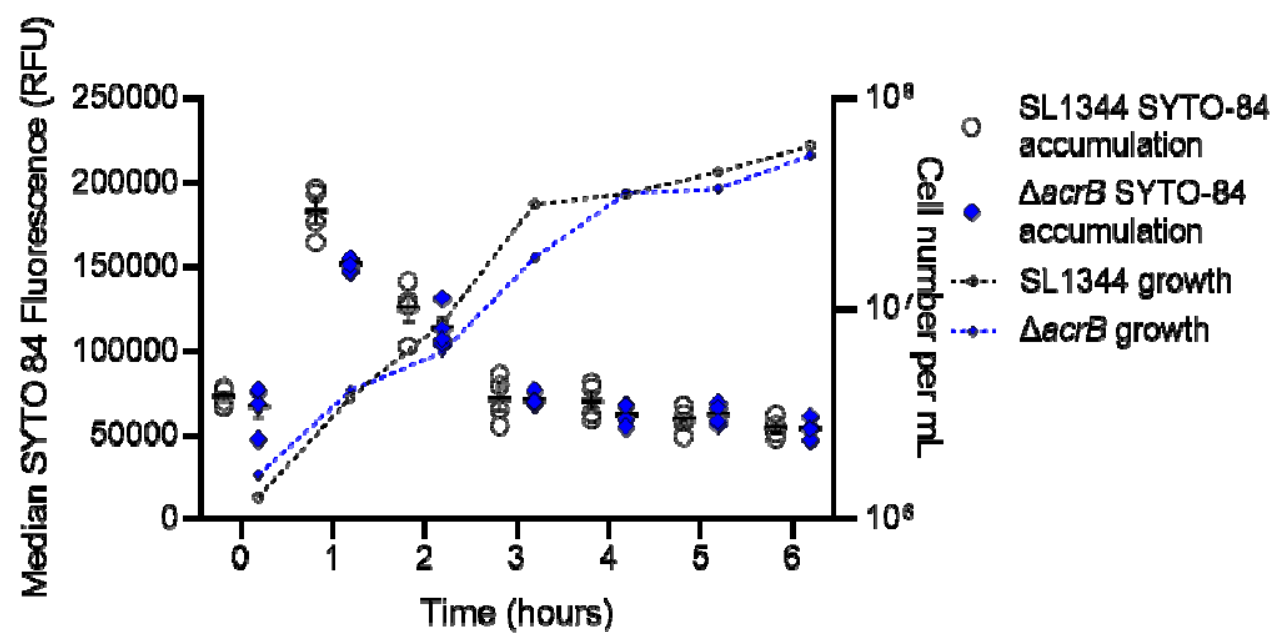

233 Figure 4 SYTO 84 accumulation in SL1344 and SL1344 $\Delta$ acrB. Median SYTO 84 fluorescence per cell in 23410,000 cells was measured every hour between 0 and 6 hours. White circles (SL1344) and blue diamonds $235(\triangle a c r B)$ represent the X-median value of SYTO 84 fluorescence in 10,000 cells within a biological replicate. 4 236 biological replicates for each strain are shown, with +/- SEM error bars. Median SYTO 84 fluorescence is plotted 237 on the left $\mathrm{Y}$-axis. Calculated cell number per $\mathrm{mL}$ values were plotted on the right $\mathrm{Y}$-axis with corresponding 238 symbols equating to strain and a dashed line to show growth of the culture. Cell numbers were based on the 239 mean of the same biological replicates and the same gated population that EtBr fluorescence was measured 240 from.

241 Ethidium bromide is a cationic dye that diffuses into cells through the $\mathrm{OM}^{32}$. LPS

242 molecules on the outer face of the outer membrane are ionically cross-linked to each

243 other by divalent cations $\left(\mathrm{Mg}^{2+}\right.$ or $\left.\mathrm{Ca}^{2+}\right)$ binding to phosphate groups in lipid $\mathrm{A}$, 
244 generating a permeability barrier. EDTA is considered a 'permeabiliser' which can

245 chelate and thus displace divalent cations, destabilising and releasing LPS $^{33}$,

246 thereby increasing the permeability of the cell to itself and other compounds ${ }^{6}$.

247 Increasing concentrations of EDTA were used to permeabilise the outer membrane

248 and assess the effect on ethidium bromide accumulation (Figure 5A). Following 1 or

2493 hours of growth, there was no significant difference in EtBr accumulation up to 100

$250 \mu \mathrm{M}$ EDTA. At $200 \mu \mathrm{M}$ and $500 \mu \mathrm{M}$ EDTA, EtBr accumulation was significantly higher,

251 suggesting that EDTA was able to make the outer membrane more permeable to

252 EtBr. At 5 hours, neither $200 \mu \mathrm{M}$ nor $500 \mu \mathrm{M}$ ETDA had any effect on the

253 accumulation of EtBr. This suggests that the Salmonella outer membrane is

254 remodelled during entry into stationary phase and becomes less reliant on cation-

255 mediated crosslinking to maintain its permeability barrier to EtBr. Indeed, both

256 Salmonella and E. coli become more resistant to CAMPs, whose mode of action

257 relies upon interaction with negative charges on the LPS, in stationary phase $\mathrm{e}^{34,35}$

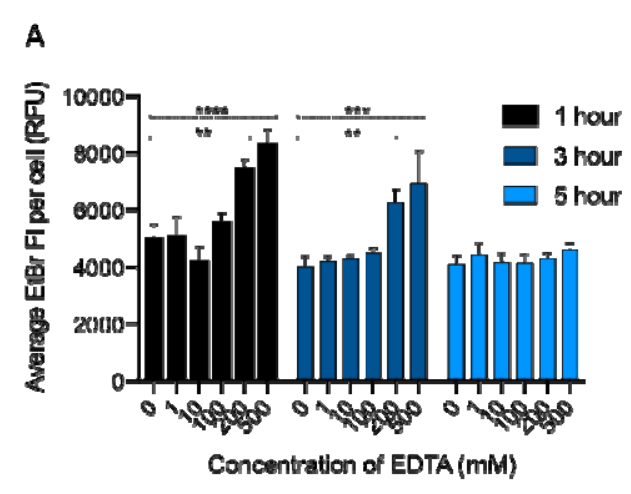

258

Figure 5 (A) EtBr accumulation in SL1344 treated with EDTA. Bars represent median EtBr fluorescence in 10,000 single cells of SL1344. EtBr accumulation was measured in the presence of increasing concentrations of $\operatorname{EDTA}(0,1,10,100,200$ and $500 \mathrm{mM})$ from a culture grown for 1 hour (black), 3 hours (dark blue) and 5 hours 
262 (light blue). Error bars show SEM from 3 biological replicates. Dashed lines above the bars with asterisks

263 represent significance value when based on a T-test compared to no EDTA added. At 1 hour, treatment with 200 and $500 \mathrm{mM}$ significantly increased $\mathrm{EtBr}$ accumulation in SL1344 with $\mathrm{p}$ values of 0.0013 ( $\left.^{* *}\right)$ and $<0.0001\left({ }^{* * * *}\right)$ respectively. At 3 hours, treatment with 200 and $500 \mathrm{mM}$ significantly increased EtBr accumulation in SL1344 with $p$ values of $0.0033\left(^{* \star}\right)$ and $0.0001\left(^{* * *}\right)$ respectively. $(B+C)$ EtBr accumulation in SL1344 $\Delta r p o S$ and $\Delta$ acrB $\Delta$ rpoS. 4 biological replicates for each strain are shown, with a short mean bar and SEM error bars. EtBr accumulation is plotted on the left $\mathrm{Y}$-axis. Calculated cell number values were plotted on right $\mathrm{Y}$-axis. Cell numbers were based on the mean of the same biological replicates and the same gated population that $\mathrm{EtBr}$ fluorescence was measured from. (B) shows SL1344 WT (individual black dots) vs $\Delta$ rpoS (blue dots). Median EtBr fluorescence per cell in 10,000 SYTO- $84^{+}$flow cytometry events was measured every hour between 0 and 6 hours. Individual symbols represent the median value of EtBr fluorescence within a biological replicate. (C) shows SL1344 $\triangle a c r B$ (black diamonds) vs $\triangle a c r B \Delta r p o S$ (green diamonds). Median EtBr fluorescence per cell in 10,000 SYTO $-84^{+}$flow cytometry events was measured every hour between 0 and 6 hours. Individual symbols represent the median value of $\mathrm{EtBr}$ fluorescence within a biological replicate. Significant differences to parent strain were measured by a two-way ANOVA and Sidak's multiple comparison test. At 3 hours, EtBr accumulation in $\triangle a c r B \Delta r p o S$ is significantly different to $\triangle a c r B$ with a $p$ value of $0.0002\left(^{* * *}\right)$.

278 A previous study found that increased SDS resistance in carbon-limited stationary

279 phase E. coli is due to decreased envelope permeability mediated by RpoS-

280 dependent and -independent mechanisms ${ }^{19}$. The role of RpoS in decreased $\mathrm{EtBr}$

281 permeability in $S$. Typhimurium was therefore investigated by construction of $\Delta r p o S$

282 mutants of SL1344 and its $\triangle a c r B$ variant.

283 Deletion of rpoS in SL1344 caused no significant difference in $\mathrm{EtBr}$ accumulation

284 (Figure 5B), although these bacteria were efflux-active so $\mathrm{EtBr}$ could be pumped

285 out. Comparison of the $\triangle a c r B$ and $\triangle r p o S \triangle a c r B$ mutants (Figure 5 C) revealed a

286 significant difference in $\mathrm{EtBr}$ accumulation only around $3 \mathrm{~h}$ growth; the $\Delta r p o S$ mutant

287 showed a delayed decrease in EtBr accumulation, although in stationary phase the

288 two strains were similar. We conclude that in S. Typhimurium, although RpoS might

289 play a role in envelope remodelling, it is not essential for generation of a low-

290 permeability envelope in stationary phase, so there are likely to be RpoS-dependent

291 and -independent pathways to achieve this phenotype. Although SDS and EDTA

292 disrupt the cell envelope in different ways (detergent disruption of lipid membranes

293 versus chelation of divalent cations), it is clear that RpoS-dependent and -

294 independent mechanisms play a role in envelope remodelling in both $E$. colli ${ }^{19}$ and $S$.

295 Typhimurium. 
297 RNAseq analysis identified several pathways likely to be involved in reduced 298 envelope permeability in S. Typhimurium

299 Given the data above did not identify a definitive mechanism by which the stationary

300 phase cell envelope displays lower permeability to $\mathrm{EtBr}$, we used RNAseq analysis

301 to identify genes and pathways that may be involved in changes to Gram-negative

302 cells as they enter stationary phase. Growing cultures of SL1344 were sampled after

3031 hour, 3 hours and 5 hours of growth and RNA was extracted and analysed by

304 GENEWIZ Inc. Comparing SL1344 at 1 hour versus 3 or 5 hours of growth, 1228

305 (26\%) and 2260 (47\%) genes were differentially expressed respectively. The data is 306 deposited with Array Express (Accession: E-MTAB-9679). Differentially-expressed

307 genes were then identified that encode proteins involved in envelope remodeling in

308 stationary phase, many of which have been shown to increase barrier function

309 (Supplementary Table S1, summarized in Figure 6).

310 Previous studies have suggested that multiple layers of the cell envelope are

311 remodeled upon entry into stationary phase ${ }^{34}$ and our RNASeq data support this; full

312 description of this dataset is in the supplementary material. Inner membrane fluidity

313 decreases with cyclopropane fatty acid incorporation ${ }^{36-38}$, mediated by upregulation

314 of cfa. Stationary phase peptidoglycan contains 3-3 (LD) rather than 4-3 (DD)

315 crosslinks $22,39,40$; relevant transpeptidases are up- and downregulated. The quantity

316 of Lpp in the OM increases (IppB is upregulated) and becomes more highly

317 crosslinked to the PG (IdtE is upregulated ${ }^{22}$ ), which has been shown to increase

318 barrier function ${ }^{41}$. OM inner leaflet cardiolipin content is known to increase ${ }^{42}$

319 potentially mediated by upregulation of $c / s B$. LPS modification pathways important in 
320 exponential phase (e.g. the pmr genes which confer CAMP resistance primarily

321 through negative charge neutralization) ${ }^{43-45}$ are downregulated whereas $I p x O$

322 (involved in myristoyl chain hydroxylation and implicated in CAMP resistance in $K$.

323 pneumonia ${ }^{46}$ ) is upregulated. Genes involved in O-antigen synthesis and chain

324 length regulation are downregulated; average O-antigen chain length increases in

325 stationary phase and O-antigen structure has been shown to influence serum

326 resistance $^{47}$ and CAMP susceptibility ${ }^{48}$. Finally, genes involved in enterobacterial

327 common antigen (ECA) synthesis are downregulated; ECA is implicated in envelope

328 integrity and bile resistance.

329 Taken together, this leads to a model (Figure 6) suggesting why the exponential

330 phase cell envelope is more susceptible to attack from various factors (EtBr, CAMPs,

331 EDTA, antibiotics). Resistance to self-mediated uptake in exponential phase is

332 provided primarily by the barrier function of LPS, comprising lipid hydrophobicity and

333 crosslinking between phosphate groups and divalent cations. The LPS takes the

334 burden because the inner layers of the envelope (PG and IM) are by necessity more

335 fluid; PG is being extensively and continually remodeled to permit growth and

336 division, and the IM is similarly fluid. The reliance on the LPS as the primary barrier

337 poses problems when antimicrobials such as EDTA and CAMPs target the

338 phosphate-cation bridges. The cell responds by shielding negative charges and

339 modifying the LPS lipid content to decrease fluidity, regulated by PmrAB.

340 In stationary phase, each layer of the envelope plays a greater role in barrier function

341 because remodeling and fluidity is less of a requirement. The lipid components of the

342 IM and OM become less fluid and the PG contains more LD-crosslinks and becomes

343 more crosslinked to the OM, further strengthening the OM permeability barrier and 
bioRxiv preprint doi: https://doi.org/10.1101/2021.05.11.443560; this version posted May 11, 2021. The copyright holder for this preprint (which

was not certified by peer review) is the author/funder, who has granted bioRxiv a license to display the preprint in perpetuity. It is made available under aCC-BY-NC-ND 4.0 International license.

344 decreasing (but not eliminating) the requirement for cation crosslinking of LPS. The

345 saccharide components of the LPS provide the outermost layer of protection. This

346 "laminated" approach shares the burden of protection and generates a strong barrier

347 against multiple chemicals which seek to enter and damage the cell, reflected by the

348 increased resistance of stationary phase cells to multiple stressors. 


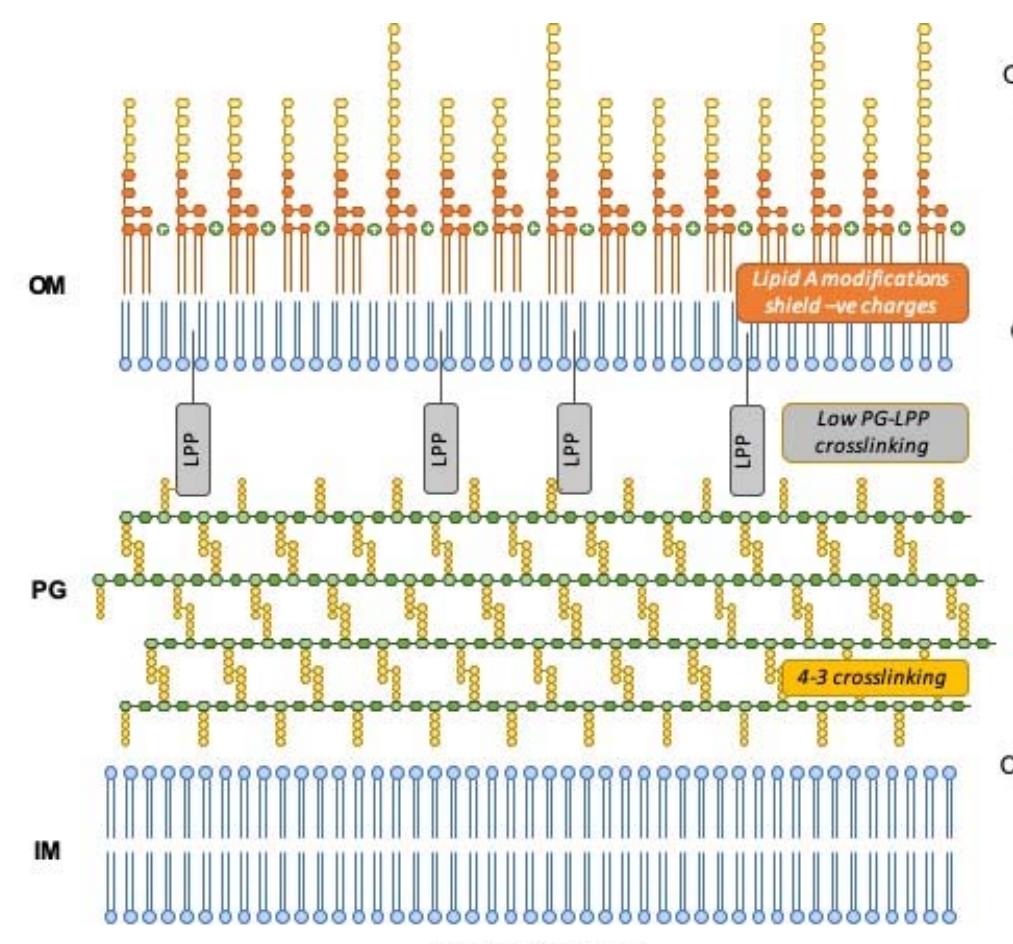

Fluid IM, dynamic PG, Lipid A \& cations are major barrier

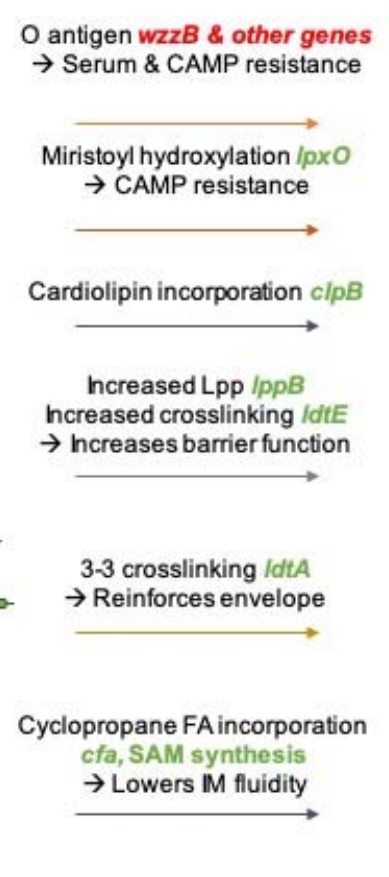

Miristoyl hydroxylation $/ p x O$
$\rightarrow$ CAMP resistance

$\longrightarrow$

$\longrightarrow$ hcreased Lpp /ppB hcreased crosslinking IdtE ncreases barrier function

3-3 crosslinking IdtA $\rightarrow$ Reinforces envelope

opropane FA incorporatio cfa, SAM synthesis $\rightarrow$ Lowers IM fluidity

Figure 6. Model showing that the differentially-expressed genes identified in the RNAseq encode proteins involved in envelope remodeling in stationary phase to increase barrier function.
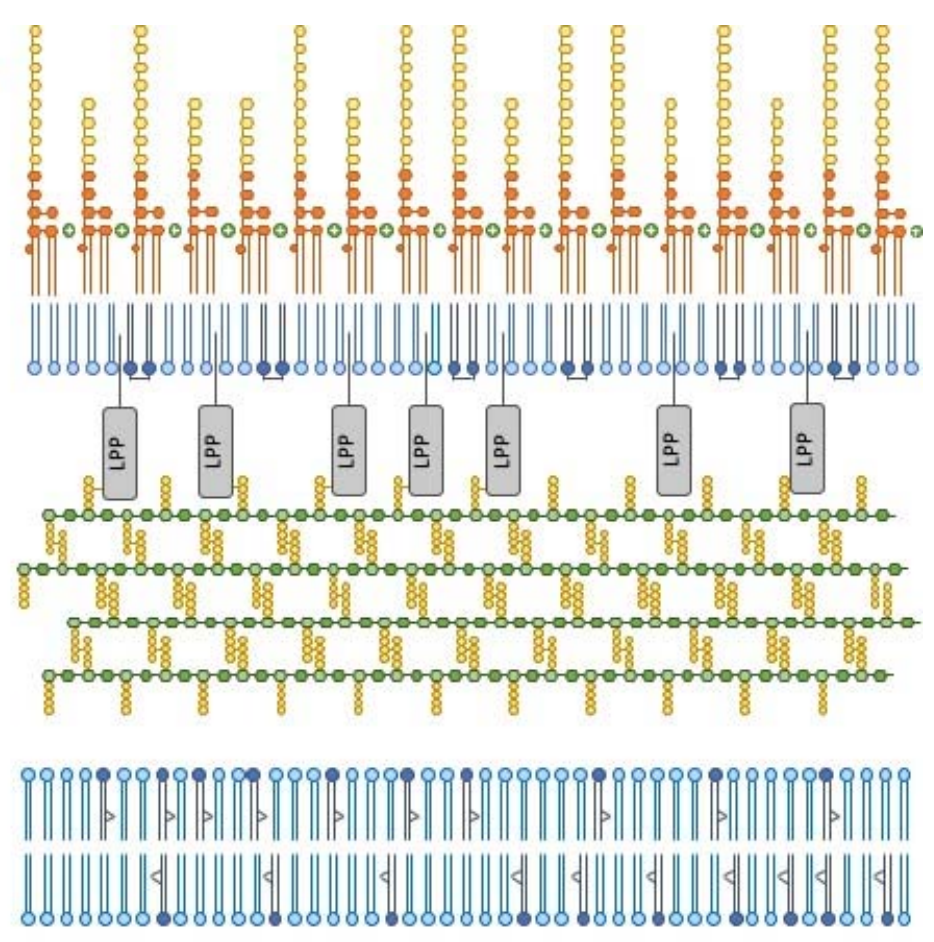

Stationary phase

Less fluid IM, reinforced PG, increased PG -OM cross links via Lpp, less barrier "load" on Lipid A 


\section{Discussion}

354 This study shows that the mechanisms that control drug accumulation are growth

355 phase dependent. In actively growing cells, efflux is fundamental to maintaining low

356 drug accumulation and subsequently survival of the bacterial population. Bacterial

357 infections are complex, and bacterial populations will often not be in a single growth

358 phase, therefore more careful consideration may be required for the most effective

359 antibiotic treatment. We have shown that stationary phase slow or non-growing cells

360 are impermeable, and that this is not due to changes in porin production but as a

361 result of membrane remodeling and increased peptidoglycan crosslinking which

362 reinforces the envelope barrier function. Treatment of chronic infections and biofilms

363 where bacterial cells are slow- or non-growing may need to be considered more

364 carefully. Successful treatment of these infections is already extremely difficult, and

365 careful consideration is already made for treatment of intrinsically impermeable

366 pathogens such as Pseudomonas aeruginosa and Acinetobacter baumanii. More

367 extensive research into the effects of an impermeable membrane on treatment

368 during infection must now be carried out.

369 Efflux pumps are only important in maintaining low drug accumulation in actively

370 growing cells which have a more permeable envelope. If an infection is actively

371 growing, it seems likely that efflux inhibitors would be effective at increasing the

372 accumulation of antibiotics within cells to potentiate their activity. However, if cells

373 are in a slow-growing or non-growing state, where membrane permeability is

374 fundamental to maintaining low drug accumulation, efflux inhibitors may not be an

375 effective treatment option. It is also possible that administering an efflux inhibitor 
376 where it has no effect on treating an infection, may also lead to the development of

377 new mechanisms of AMR.

379 Materials and Methods

380 Strains and growth conditions

381 Unless otherwise stated, all experiments use Salmonella enterica serovar

382 Typhimurium (hereafter named $S$. Typhimurium ${ }^{49}$ ) SL1344. The $\Delta$ acrB and $\Delta 4 \mathrm{PAP}$

383 strains ( $\triangle$ acrA $\Delta$ acrE $\Delta m d s A \Delta m d t A)$ have been previously published ${ }^{50,51}$. SL1344

$384 \Delta o m p F$ and $\triangle o m p C$ strains were constructed for this study using the Datsenko and

385 Wanner method of gene deletion ${ }^{52}$. Transcriptional reporter constructs were made by

386 fusing the promoter of each efflux pump gene to $g f p$ in the pMW82 plasmid ${ }^{53}$. These

387 plasmids were transformed into SL1344 and SL1344 $\Delta$ acrB. E. coli MG1655

$388 \Delta a c r B^{15}$, P. aeruginosa PA01 $\Delta m e x A^{54}$ and $K$. pneumoniae ecl8 acrB::Gm ${ }^{17}$ were

389 also used as part of this study and are previously published.

390 Unless otherwise stated, LB (Sigma) was used as growth medium for all assays.

391 One assay used MOPs minimal media (Teknova) which was supplemented with 400

$392 \mathrm{mg} / \mathrm{L}$ histidine.

393 Chromosomal insertion of gfp downstream of acrB to produce SL1344 AcrB-

394 GFP

395 To measure the protein level of AcrB in S. Typhimurium, a gene encoding a

396 monomeric super-folder GFP (msfGFP) was inserted downstream of acrB on the

397 chromosome to produce an AcrB-msfGFP fusion protein. This strain was created 
398 using the msfGFP from the pET GFP LIC cloning vector (u-msfGFP) which was a gift

399 from Scott Gradia (Addgene plasmid \# 29772 ; http://n2t.net/addgene:29772 ;

400 RRID:Addgene_29772). Strain construction was based on the method used by

401 Bergmiller et al. (2017) in E. coli ${ }^{27}$ where the codon optimised polylinker was used.

402 Using restriction and ligation, the aph gene was inserted into pET LIC vector

403 (u-msfGFP), so that strains containing the plasmid could be selected for. Using this

404 plasmid as template, gfp and aph were inserted into the chromosome downstream of

405 acrB in SL1344 to produce a protein fusion strain.

407 Flow cytometry assay

408 The flow cytometric EtBr accumulation assay has been previously described ${ }^{17}$. Here

409 this method was used to measure accumulation in samples from the same culture at

410 different timepoints during batch culture. Briefly, cultures were grown at $37^{\circ} \mathrm{C}$

411 overnight in $5 \mathrm{~mL}$ of LB and sub-cultured at $4 \%$ into fresh LB. A sample was taken

412 at 0 hours and then every hour for 6 hours during growth. At each hour, sample

413 volume was adjusted such that approximately $10^{7}$ cells were harvested and re-

414 suspended in 1 x Hepes Buffered Saline (5X HBS; Alfa Aesar). Cells were washed

415 and resuspended in $1 \mathrm{~mL}$ HBS. $100 \mu \mathrm{L}$ of cell suspension was then further diluted

416 into $500 \mu \mathrm{L}$ HBS and SYTO ${ }^{\mathrm{TM}} 84$ (Thermo Fisher Scientific) and ethidium bromide

417 added to give final concentrations of $10 \mu \mathrm{M}$ and $100 \mu \mathrm{M}$ respectively. Samples were

418 incubated for 10 minutes before measuring accumulation by flow cytometry. Flow

419 cytometry settings and emission filters were used from Whittle et al ${ }^{17}$. Briefly, The

420 SYTO 84 fluorescence emission was collected in the YL1-H channel (585/16 nm)

421 using a $561 \mathrm{~nm}$ yellow laser and used to differentiate cells from acellular material. 
422 EtBr fluorescence was collected using the BL3-H channel $(695 / 40 \mathrm{~nm})$ using a 488

$423 \mathrm{~nm}$ blue laser. SYTO 84 accumulation measurements (Figure 4), was not a

424 repeated experiment but data was re-analysed from $\mathrm{EtBr}$ accumulation assays and

425 therefore fluorescence emission was collected in the YL1-H channel $(585 / 16 \mathrm{~nm})$

426 using a $561 \mathrm{~nm}$ yellow laser. Nile Red accumulation was measured as previously

427 described ${ }^{17}$. In these experiments SYTO $9(10 \mu \mathrm{M}$; Thermo Fisher Scientific) was

428 used to differentiate cells from acellular particles using the BL2-H channel. Nile red

429 has an excitation of $549 \mathrm{~nm}$ and emission of $628 \mathrm{~nm}$ in the presence of

430 phospholipids, and in a neutral lipid environment (tryglycerides), the fluorescence

431 shifts to ex/em of $510 / 580 \mathrm{~nm}^{31}$. Nile red fluorescence was excited using the yellow

432 laser and detected using the $\mathrm{YL1}-\mathrm{H}$ channel for orange fluorescence ${ }^{17}$.

\section{Flow cytometry assay in the presence of EDTA}

434 Growing culture samples were taken at 1, 3 and 5 hours as above. Samples were

435 made with varying concentrations of EDTA $(0 \mu \mathrm{M}, 1 \mu \mathrm{M}, 10 \mu \mathrm{M}, 100 \mu \mathrm{M}, 200 \mu \mathrm{M}$

436 and $500 \mu \mathrm{M})$ in $500 \mu \mathrm{L}$ HBS. These concentrations of EDTA increased the final

437 volume of the sample because the stock concentration was limited by solubility.

438 Dyes were then added but volume added was adjusted to maintain the final

439 concentration stated above. Once the dyes were added, $100 \mu \mathrm{L}$ of cell suspension

440 was added and cells were incubated for 10 minutes at room temperature. Samples

441 were then analysed by flow cytometry.

\section{Whole population transcription analysis}

443 Overnight cultures containing pMW82 transcriptional reporter plasmids were diluted

$4441: 10000$ in MOPs minimal media, supplemented with $50 \mu \mathrm{g} / \mathrm{ml}$ ampicillin. $\mathrm{OD}_{600}$ and 
445 GFP fluorescence were measured every 30 minutes for 12 hours using a Fluostar

446 Omega (BMG labtech) incubated at $37^{\circ} \mathrm{C} . \mathrm{OD}_{600}$ and GFP fluorescence were

447 measured, and a minimal media only control subtracted from the data. SL1344

448 autofluorescence was removed by subtracting SL1344 fluorescence from that of

449 pMW82 strains. GFP fluorescence divided by $\mathrm{OD}_{600}$ was used as a measurement to

450 disregard cell density across growth.

\section{Efflux assay}

452 Efflux assays were carried out as previously described as previously ${ }^{14}$. This assay

453 measures direct efflux activity of a population of cells by pre-loading cells with a

454 fluorescent efflux substrate in the presence of the proton motive force inhibitor,

455 CCCP, and re-energising cells with glucose to measure the decrease in fluorescence

456 as substrates leave the cells. Briefly, overnight cultures of SL1344 and SL1344

$457 \Delta a c r B$ were sub-cultured into fresh LB and then grown for 5 hours at $37^{\circ} \mathrm{C}$. At the 1 ,

4583 and 5 -hour time points, $10 \mathrm{~mL}$ of culture was taken and the $\mathrm{OD}_{600}$ measured. The

459 harvested cell pellet was then resuspended in phosphate buffer containing $\mathrm{MgCl}_{2}$

460 buffer and each strain adjusted to the same $\mathrm{OD}_{600}$.

\section{RNAseq}

462 The transcriptome of SL1344 and SL1344 $\triangle$ acrB were analysed at different time

463 points during growth (1, 3 and 5 hours). There were 4 replicates of each strain.

464 MOPS minimal media was inoculated at $4 \%$ with overnight cultures. Cultures were

465 incubated at $37^{\circ} \mathrm{C}$, shaking for 5 hours. At 1 hour, $5 \mathrm{~mL}$ of culture was centrifuged at

$4663500 \times \mathrm{g}$ for 5 minutes at room temperature to harvest the cells. The supernatant

467 was removed and the pellet was snap frozen. At the 3 and 5 - hour time points, only 1 
$468 \mathrm{~mL}$ of culture was harvested and snap frozen. GENEWIZ Inc. carried out the RNA

469 extraction, quality control, library preparation, sequencing and bioinformatic analysis.

470 Briefly, total RNA was extracted from S. Typhimurium cell pellets using RNeasy Plus

471 Universal kit (Qiagen), and RNA quality control was carried out using Qubit 2.0

472 Fluorometer to measure total RNA concentration and Agilent TapeStation to produce

473 an RNA integrity number (RIN) and a DV200 score. To remove rRNA, the ribozero

474 Removal Kit was used (Illumina). The NEBNext Ultra II RNA Library Prep Kit

475 (Illumina) was used for library preparation, following the manufacturer's protocol. For

476 library preparation, cDNA was synthesised, end repaired and adenylated at the 3'

477 ends. Universal adapters were ligated to cDNA and library enrichment was carried

478 out using limited cycle PCR. Sequencing was carried out using Illumina HiSeq 4000.

479 Bioinformatic data analysis was carried out by GENEWIZ Inc. Trimmed reads were

480 mapped to the SL1344 reference genome FQ312003 using the Bowtie2 aligner.

481 Unique gene hit counts were calculated by using feature Counts from the Subread

482 package. All statistical analysis was performed using R. With the package, DESeq2,

483 a comparison of gene expression between the groups of samples was performed.

484 The Wald test was used to generate $p$-values and Log2 fold changes. Data is

485 accessible on ArrayExpress with the accession code E-MTAB-9679.

\section{Conflict of Interest Statement}

488 The authors declare that the research was conducted in the absence of any

489 commercial or financial relationships that may be considered as a conflict of interest.

\section{Authors Contributions Statement}


491 JMAB, TWO and EEW designed these assays. GFP transcriptional reporter strains

492 were constructed by ET. EEW and HM performed experiments to obtain samples for

493 RNAseq. EEW performed all other experiments. EEW analysed all data. RNAseq

494 data was analysed by EEW and TWO. This manuscript was written by EEW, JMAB,

495 TWO and MAW.

496 Funding

497 EEW was funded by the AAMR Wellcome Trust DTP grant $108876 / B / 15 / Z$ at the

498 University of Birmingham. JMAB and HM were funded by BBSRC grant

499 BB/M02623X/1 (David Phillips Fellowship to JMAB).

\section{$501 \quad$ References}

502 1. Nikaido, H. Outer membrane barrier as a mechanism of antimicrobial

503 resistance. Antimicrobial Agents and Chemotherapy (1989)

504 doi:10.1128/AAC.33.11.1831.

505 2. Pratt, L. A., Hsing, W., Gibson, K. E. \& Silhavy, T. J. From acids to osmZ:

$506 \quad$ Multiple factors influence synthesis of the OmpF and OmpC porins in

507 Escherichia coli. Molecular Microbiology (1996) doi:10.1111/j.1365-

$508 \quad$ 2958.1996.tb02532.x.

509 3. Dupont, H. et al. Structural alteration of OmpR as a source of ertapenem

510 resistance in a CTX-M-15-producing Escherichia coli O25b:H4 sequence type

$511 \quad 131$ clinical isolate. Antimicrob. Agents Chemother. (2017)

512 doi:10.1128/AAC.00014-17. 
513 4. Dé, E. et al. A new mechanism of antibiotic resistance in Enterobacteriaceae

514 induced by a structural modification of the major porin. Mol. Microbiol. (2001)

$515 \quad$ doi:10.1046/j.1365-2958.2001.02501.x.

516 5. Bajaj, H. et al. Molecular Basis of filtering carbapenems by porins from

$517 \quad$ Blactam-resistant clinical strains of Escherichia coli. J. Biol. Chem. (2016)

518 doi:10.1074/jbc.M115.690156.

519 6. Hancock, R. E. W. \& Bell, A. Antibiotic uptake into gram-negative bacteria. in

$520 \quad$ Current Topics in Infectious Diseases and Clinical Microbiology (1989).

521 doi:10.1007/978-3-322-86064-4_5.

522 7. Hancock, R. E. W., Raffle, V. J. \& Nicas, T. I. Involvement of the outer

523 membrane in gentamicin and streptomycin uptake and killing in Pseudomonas

524 aeruginosa. Antimicrob. Agents Chemother. (1981) doi:10.1128/AAC.19.5.777.

525 8. Delcour, A. H. Outer membrane permeability and antibiotic resistance.

526 Biochimica et Biophysica Acta - Proteins and Proteomics (2009)

527 doi:10.1016/j.bbapap.2008.11.005.

528 9. Hancock, R. E. Alterations in outer membrane permeability. Annual review of

529 microbiology (1984) doi:10.1146/annurev.mi.38.100184.001321.

530 10. Hassan, K. A. et al. Pacing across the membrane: the novel PACE family of

531 efflux pumps is widespread in Gram-negative pathogens. Res. Microbiol.

$532 \quad$ (2018) doi:10.1016/j.resmic.2018.01.001.

533 11. Blair, J. M. A. et al. Expression of homologous RND efflux pump genes is

534 dependent upon AcrB expression: Implications for efflux and virulence inhibitor 
535 design. J. Antimicrob. Chemother. (2015) doi:10.1093/jac/dku380.

536 12. Webber, M. A. \& Piddock, L. J. V. Absence of mutations in marRAB or soxRS

537 in acrB-overexpressing fluoroquinolone-resistant clinical and veterinary

538 isolates of Escherichia coli. Antimicrob. Agents Chemother. (2001)

539 doi:10.1128/AAC.45.5.1550-1552.2001.

540 13. Chowdhury, N. et al. Identification of AcrAB-TolC Efflux Pump Genes and

541 Detection of Mutation in Efflux Repressor AcrR from Omeprazole Responsive

$542 \quad$ Multidrug-Resistant Escherichia coli Isolates Causing Urinary Tract Infections.

$543 \quad$ Microbiol. Insights (2019) doi:10.1177/1178636119889629.

544 14. Smith, H. E. \& Blair, J. M. A. Redundancy in the periplasmic adaptor proteins

545 AcrA and AcrE provides resilience and an ability to export substrates of

546 multidrug efflux. J. Antimicrob. Chemother. (2014) doi:10.1093/jac/dkt481.

547 15. Wang-Kan, X. et al. Lack of AcrB efflux function confers loss of virulence on

$548 \quad$ Salmonella enterica serovar typhimurium. MBio (2017)

$549 \quad$ doi:10.1128/mBio.00968-17.

550 16. Blair, J. M. A. \& Piddock, L. J. V. How to measure export via bacterial

551 multidrug resistance efflux pumps. MBio (2016) doi:10.1128/mBio.00840-16.

552 17. Whittle, E. E. et al. Flow Cytometric Analysis of Efflux by Dye Accumulation.

$553 \quad$ Front. Microbiol. (2019) doi:10.3389/fmicb.2019.02319.

554 18. Bailey, A. M., Webber, M. A. \& Piddock, L. J. V. Medium plays a role in

555 determining expression of $a c r B$, marA, and soxS in Escherichia coli.

556 Antimicrob. Agents Chemother. (2006) doi:10.1128/AAC.50.3.1071- 
1074.2006.

558

559

560

561

562

563

564

565

566

567

568

569

570

571

572

573

574

19. Mitchell, A. M., Wang, W. \& Silhavy, T. J. Novel RpoS-dependent mechanisms strengthen the envelope permeability barrier during stationary phase. J. Bacteriol. (2017) doi:10.1128/JB.00708-16.

20. Allen, R. J. \& Scott, G. K. Biosynthesis and turnover of outer-membrane proteins in Escherichia coli ML308-225. Biochem. J. 182, 407-412 (1979).

21. WENSINK, J., GILDEN, N. \& WITHOLT, B. Attachment of Lipoprotein to the Murein of Escherichia coli. Eur. J. Biochem. (1982) doi:10.1111/j.14321033.1982.tb06479.x.

22. Glauner, B., Holtje, J. V. \& Schwarz, U. The composition of the murein of Escherichia coli. J. Biol. Chem. (1988) doi:10.1016/s0021-9258(19)81481-3.

23. El-Khani, M. A. \& Stretton, R. J. Effect of growth medium on the lipid composition of log and stationary phase cultures of Salmonella typhimurium. Microbios (1981).

24. Grogan, D. W. \& Cronan, J. E. Cyclopropane ring formation in membrane lipids of bacteria. Microbiol. Mol. Biol. Rev. (1997) doi:10.1128/.61.4.429441.1997.

25. Mengin-Lecreulx, D. \& Van Heijenoort, J. Effect of growth conditions on peptidoglycan content and cytoplasmic steps of its biosynthesis in Escherichia coli. J. Bacteriol. (1985) doi:10.1128/jb.163.1.208-212.1985.

26. Chai, Q., Webb, S. R., Wang, Z., Dutch, R. E. \& Wei, Y. Study of the degradation of a multidrug transporter using a non-radioactive pulse chase 
method. Anal. Bioanal. Chem. (2016) doi:10.1007/s00216-016-9871-7.

580 27. Bergmiller, T. et al. Biased partitioning of the multidrug efflux pump AcrAB-

581 TolC underlies long-lived phenotypic heterogeneity. Science (80-. ). (2017)

582 doi:10.1126/science.aaf4762.

583 28. Pagès, J. M. \& Amaral, L. Mechanisms of drug efflux and strategies to combat

29. Pagès, J. M., Masi, M. \& Barbe, J. Inhibitors of efflux pumps in Gram-negative them: Challenging the efflux pump of Gram-negative bacteria. Biochimica et Biophysica Acta - Proteins and Proteomics (2009) doi:10.1016/j.bbapap.2008.12.011.

30. Nikaido, H. Small Things Considered: The Limitations of LB Medium. Small Things Considered (2009).

31. Greenspan, P. \& Fowler, S. D. Spectrofluorometric studies of the lipid probe, nile red. J. Lipid Res. (1985) doi:10.1016/S0022-2275(20)34307-8.

594 32. Murata, T., Tseng, W., Guina, T., Miller, S. I. \& Nikaido, H. PhoPQ-mediated regulation produces a more robust permeability barrier in the outer membrane of Salmonella enterica serovar typhimurium. J. Bacteriol. 189, (2007). Biochem. Biophys. Res. Commun. (1965) doi:10.1016/0006-291X(65)90191-9. 
601 is $\operatorname{RpoS}(\sigma(S))$ independent and occurs through both phoP-dependent and -

602 independent pathways. J. Bacteriol. 178, (1996).

603 35. Agrawal, A., Rangarajan, N. \& Weisshaar, J. C. Resistance of early stationary

604 phase E. coli to membrane permeabilization by the antimicrobial peptide

605 Cecropin A. Biochim. Biophys. Acta - Biomembr. 1861, (2019).

606 36. Bianco, C. M., Fröhlich, K. S. A. \& Vanderpoola, C. K. Bacterial cyclopropane

607 fatty acid synthase mRNA is targeted by activating and repressing small

608 RNAs. J. Bacteriol. (2019) doi:10.1128/JB.00461-19.

609 37. Qi, Y., Liu, H., Chen, X. \& Liu, L. Engineering microbial membranes to

610 increase stress tolerance of industrial strains. Metabolic Engineering (2019)

$611 \quad$ doi:10.1016/j.ymben.2018.12.010.

612 38. Huisman, G. W., Siegele, D. a, Zambrano, M. M. \& Kolter, R. Morphological

613 and physiological changes during stationary phase. Escherichia coli

614 Salmonella Cell. Mol. Biol. (1996).

615 39. Morè, N. et al. Peptidoglycan remodeling enables Escherichia coli to survive

616 severe outer membrane assembly defect. MBio (2019)

617 doi:10.1128/mBio.02729-18.

618 40. Magnet, S., Dubost, L., Marie, A., Arthur, M. \& Gutmann, L. Identification of the

619 L,D-transpeptidases for peptidoglycan cross-linking in Escherichia coli. J.

$620 \quad$ Bacteriol. 190, (2008).

621 41. Peters, K. et al. Copper inhibits peptidoglycan LD-transpeptidases suppressing

$622 \quad \beta$-lactam resistance due to bypass of penicillin-binding proteins. Proc. Natl. 
Acad. Sci. U. S. A. (2018) doi:10.1073/pnas.1809285115.

624 42. Hiraoka, S., Matsuzaki, H. \& Shibuya, I. Active increase in cardiolipin synthesis

625 in the stationary growth phase and its physiological significance in Escherichia 626 coli. FEBS Lett. 336, (1993).

627 43. Raetz, C. R. H., Reynolds, C. M., Trent, M. S. \& Bishop, R. E. Lipid a modification systems in gram-negative bacteria. Annual Review of Biochemistry (2007) doi:10.1146/annurev.biochem.76.010307.145803.

44. May, J. F. \& Groisman, E. A. Conflicting roles for a cell surface modification in Salmonella. Mol. Microbiol. 88, (2013).

45. Agrawal, A. \& Weisshaar, J. C. Effects of alterations of the E. coli lipopolysaccharide layer on membrane permeabilization events induced by Cecropin A. Biochim. Biophys. Acta - Biomembr. 1860, (2018).

46. Llobet, E. et al. Deciphering tissue-induced Klebsiella pneumoniae lipid a structure. Proc. Natl. Acad. Sci. U. S. A. (2015) doi:10.1073/pnas.1508820112.

47. Bravo, D. et al. Growth-phase regulation of lipopolysaccharide O-antigen chain length influences serum resistance in serovars of Salmonella. J. Med. Microbiol. 57, (2008).

48. Ricci, V., Zhang, D., Teale, C. \& Piddock, L. J. V. The o-antigen epitope governs susceptibility to colistin in Salmonella enterica. MBio 11, (2020).

642 49. Brenner, F. W., Villar, R. G., Angulo, F. J., Tauxe, R. \& Swaminathan, B. 
645 50. Eaves, D. J., Ricci, V. \& Piddock, L. J. V. Expression of $a c r B$, $a c r F$, acrD,

646 marA, and soxS in Salmonella enterica Serovar Typhimurium: Role in Multiple

647 Antibiotic Resistance. Antimicrob. Agents Chemother. (2004)

648 doi:10.1128/AAC.48.4.1145-1150.2004.

649 51. McNeil, H. E. et al. Identification of binding residues between periplasmic

650 adapter protein (PAP) and RND efflux pumps explains PAP-pump promiscuity

651 and roles in antimicrobial resistance. PLoS Pathog. (2019)

652 doi:10.1371/JOURNAL.PPAT.1008101.

653 52. Datsenko, K. A. \& Wanner, B. L. One-step inactivation of chromosomal genes

654 in Escherichia coli K-12 using PCR products. Proc. Natt. Acad. Sci. U. S. A.

$655 \quad$ (2000) doi:10.1073/pnas.120163297.

656 53. Bumann, D. \& Valdivia, R. H. Identification of host-induced pathogen genes by differential fluorescence induction reporter systems. Nat. Protoc. (2007)

658 doi:10.1038/nprot.2007.78.

659 54. Held, K., Ramage, E., Jacobs, M., Gallagher, L. \& Manoil, C. Sequence660 verified two-allele transposon mutant library for Pseudomonas aeruginosa 661 PAO1. J. Bacteriol. (2012) doi:10.1128/JB.01479-12. 